\title{
Association between RT-PCR Ct values and COVID-19 new daily cases: a multicenter cross-sectional study
}

\author{
Abdulkarim Abdulrahmann, ${ }^{1,2}$, Saad I. Mallah ${ }^{3},{\text { Abdulla Alawadhi, }{ }^{1,4}, \text { Simone Perna }}^{5}$, \\ Essam M. Janahi ${ }^{6}$, Manaf M AlQahtani1,3,4 \\ ${ }^{1}$ The National Taskforce for Combating the Coronavirus (COVID-19), Kingdom of Bahrain; \\ ${ }^{2}$ Mohammed Bin Khalifa Cardiac Centre, Kingdom of Bahrain; \\ ${ }^{3}$ Royal College of Surgeons in Ireland - Bahrain, Kingdom of Bahrain; \\ ${ }^{4}$ Bahrain Defence Force Hospital, Kingdom of Bahrain; \\ ${ }^{5}$ Department of Biology, College of Science, University of Bahrain, Sakhir Campus, Kingdom of Bahrain; \\ IIndependent Researcher, Building 1848, Road 7542, Al Janabiyah 0575, Northern Governorate, Bahrain
}

Article received 1 May, 2021; accepted 16 July, 2021

\section{SUMMARY}

Proactive prediction of the epidemiologic dynamics of viral diseases and outbreaks of the type of COVID-19 has remained a difficult pursuit for scientists, public health researchers, and policymakers. It is unclear whether RT-PCR Cycle Threshold $(\mathrm{Ct})$ values of COVID-19 - or any other virus - as indicator of viral load, could represent a possible predictor for underlying epidemiologic changes on a population level. The study objective is thus to investigate whether population-wide changes in SARS-CoV-2 RT-PCR Ct values over time are associated with the daily fraction of positive COVID-19 tests. In addition, this study analyses the factors that could influence RT-PCR Ct values. A retrospective cross-sectional study was conducted on 63,879 patients from May 4, 2020 to September 30, 2020, in all COVID-19 facilities in the Kingdom of Bahrain. Data collected included number of tests and newly diagnosed cases, as well as Ct values, age, sex nationality, and symptomatic status.

$\mathrm{Ct}$ values were found to be negatively and very weakly correlated with the fraction of daily positive tests in the population $\mathrm{r}=-0.06$ (CI 95\%: $-0.06 ;-0.05 ; \mathrm{p}=0.001)$. The R-squared for the regression model (adjusting for age and number of daily tests) showed an accuracy of $45.3 \%$. Ct Values showed an association with nationality $(p=0.012)$. After the stratification, the association between $\mathrm{Ct}$ values and the fraction of daily positive cases was only maintained for the female sex and Bahraini-nationality. Symptomatic presentation was significantly associated with lower $\mathrm{Ct}$ values (higher viral loads). Ct values do not show any correlation with age $(\mathrm{p}=0.333)$ or $\operatorname{sex}(\mathrm{p}=0.522)$.

We report one of the first and largest studies to investigate the epidemiologic associations of $\mathrm{Ct}$ values with COVID-19. Although changes in Ct values showed a moderate association with daily cases, our results indicate that it may not be as predictive within a simple model. More population studies and models from global cohorts are necessary.

Keywords: COVID-19; SARS-CoV-2; viral load; RT-PCR Ct value; forecast.

\section{INTRODUCTION}

oronavirus Disease 2019 (COVID-19) has ravaged the world over a short period of time. When discussing the epidemiology of a virus, a

Corresponding author

Manaf M AlQahtani

E-mail: mqahtani@rcsi-mub.com thorough understanding of its transmission is necessary. SARS-CoV-2, the causative agent of COVID-19, primary mode of transmission is through respiratory droplets and direct or indirect contact routes [1]. Indirect contact through fomites may also be a source of infection when transferred from surfaces that have been contaminated with infected droplets, to the mouth, nose, or eyes. Although the epidemiology of SARS-CoV-2 doesn't 
support airborne transmission in public settings, an increasing number of publications are pointing to such route. The exact role of asymptomatic and pre-symptomatic transmission in the COVID-19 outbreak is not fully comprehended as of yet, but is believed to have an important contribution to the viruses' rapid spread. SARS-CoV-2 RNA may be detected 1-3 days prior to symptom onset. The viral load is highest around the day of symptom onset, followed by a gradual decline over time. RT-PCR positivity may last for 1-2 weeks in the case of asymptomatic patients, and up to 3 weeks for those with mild to moderate disease [2].

It is important to differentiate between viral load and infectious dose, and the inter-related implications of both. The infectious dose of a virus is the dose at which if a person is exposed to it would be sufficient to cause an infection. On the other hand, viral load is the amount of virus particles that the infected patient carries in their system. The higher the viral load of a patient, the more virus is shed, which therefore leads to a higher chance of others being exposed to the infectious dose needed to acquire the disease. As with viruses in general, the viral load of COVID-19 patient can be deduced from the Cycle threshold $(\mathrm{Ct})$ value of the RTPCR test conducted on the obtained sample. The RT-PCR test amplifies the viral RNA from the patient's sample until it is at a detectable concentration that exceeds the threshold value. The number of cycles necessary for that to take place is known as the $\mathrm{Ct}$ value. Thus, the lower the $\mathrm{Ct}$ value of a patient's sample, the higher the viral load, whereas the higher it is the lower the viral load [3]. According to one study, patients with severe symptoms presented with 60 times higher viral load and prolonged viral shedding than patients with mild symptoms, which may indicate a role for viral load not only in diagnosis, but rather prognosis and transmission/contagiousness. Studies showed that lower $\mathrm{Ct}$ values were associated with a higher probability of a positive viral culture. In one study, infectivity (defined as growth in cell culture) was significantly reduced when RT-PCR $\mathrm{Ct}$ values were over 24 [4]. On the other hand, La Scola et al. (2020) have reported that patients with Ct values $\geq 34$ are non-infectious and can be discharged from hospitals or care centers [5]. The implications of $\mathrm{Ct}$ values or viral load, if any, on the collective population from an epidemiologic perspective has not been adequately studied yet.
Proactive forecasting of the disease's epidemiology has remained a distant desire and puzzling enigma for scientists, public health researchers, and policymakers. Unlike previous outbreaks, the impact of the COVID-19 pandemic has transcended the medical and clinical setting, bringing forth major global disruption to public life in the social and professional settings, as well as serious economic repercussions on national and global levels [6]. Countries' deployment of their health resources and response preparedness has also been a source of concern, due to the unexpected nature and trajectorial development of the pandemic [7]. For these reasons, the prediction or forecasting of an upcoming surge in COVID-19 cases is an important item on the agenda of the scientific community and decision-makers. The daily number of positive cases on a global level has been widely variable. Forecasting attempts have been utilized to a fair degree of accuracy so far. The US Centers for Disease Control and Prevention (CDC) publishes multiple forecasts modelled by independent institutions, predicting the daily number of new cases in the USA. The models use a variety of data types besides those directly related to COVID-19, including demographic and mobility data, as well as different methods and estimates of the impact of public health interventions/policies and how these levels of precautions will change [8]. The previous forecasts when compared to actual data do fairly well in terms of general trend and values to be expected [9]. However; the multivariable models are fairly complex and may not be readily available for use in other countries.

Therefore, this study objective is to investigate whether population-wide changes in SARS-CoV-2 RT-PCR Ct values over time, are associated with the number of new COVID-19 cases in the future, as a pilot study to the development of an epidemiologic model. Our hypothesis is based on that higher viral load indicates increased viral shedding, increased risk of exposure to an infectious dose, and thus a higher degree of transmissibility. Hence, a decrease in Ct value could predict an increase or a surge in the number of positive cases. In order to develop a predictive model, other epidemiological factors that may be associated with $\mathrm{Ct}$ values, and thus must be fed into the model, were also investigated. The ability to predict population changes in the number of COVID-19 positive cases ahead of time using a widely avail- 
able and simple metric can be very helpful. Public health measures and policies can be proactively tailored to control and manage the outbreak in an informed and prophylactic manner.

\section{PATIENTS AND METHODS}

Study population $\mathcal{E}$ data collection

A retrospective study was conducted on 63,879 patients from May 4, 2020 to September 30, 2020, in all COVID-19 facilities in the Kingdom of Bahrain. Data on the number of tests and newly diagnosed cases were obtained from the National COVID-19 Task force's centralized database. Data collected included age, sex, nationality, symptomatic status. Ct Values from newly diagnosed cases in this time period were obtained from the corresponding medical records.

\section{Diagnosis and SARS-Cov-2 RNA amplification}

All cases were diagnosed as COVID-19 based on RT-PCR tests of nasopharyngeal samples. The nasopharyngeal samples were transferred to a viral transport media immediately after collection and transported to a COVID-19 laboratory for testing. Diagnosed cases were a heterogeneous sample of tested patients, including symptomatic individuals, close contacts, travelers, healthcare worker screening, and random population testing. The RT-PCR tests were conducted using different kits: Thermo Fisher Scientific (Waltham, MA) TaqPath 1-Step RT-qPCR Master Mix, CG (catalog number A15299), SuperScript ${ }^{\mathrm{TM}}$ III Platinum ${ }^{\mathrm{TM}}$ OneStep qRT-PCR Kit (catalog number 11732020), LightMix1 Modular SARS-CoV (COVID-19) (TIB MOLBIOL, Berlin, Germany). The RT-PCR was conducted on the Applied Biosystems (Foster City, CA) 7500 Fast Dx RealTime PCR Instrument, LightCycler $^{\circledR} 480$ Instrument II (Roche Molecular Systems, Inc). The assay used targeted the E gene. If positive, the sample was confirmed by RdRP and $\mathrm{N}$ genes. The $\mathrm{E}$ gene $\mathrm{Ct}$ value was reported and used in this study. Ct Values $>40$ were considered negative.

\section{RT-qPCR analytical validation}

Positive and negative controls were included for quality control purposes. Linear range was determined using serial $\log _{10}$ dilutions of standard RNA and was established between $3.5 \times 10^{3}$ to $3.5 \times 10^{12}$ copies of $\mathrm{RNA} / \mathrm{mL}$, with amplification efficiency of $87 \%$ and with a regression $R^{2}$ value higher than 0.99 .

\section{Data handling and statistical analysis}

Statistical analyses were performed using SPSS 25.0 (SPSS, Inc., Chicago, IL, USA). The sample size was not calculated as this was a pilot study. Mean and standard deviation (SD) for all variables were given as descriptive statistics. Continuous variables were calculated between groups by means and SDs and frequencies. Categorical variables were reported as frequencies and percentages. The data was grouped into a daily set consisting of 150 days, and a weekly set, consisting of 21 equal weeks starting on May $4^{\text {th }}$ and ending on September $27^{\text {th }}$.

According to the Kolmogorov-Smirnov normality test, all variables were normally distributed. The main endpoint was assessed with the Pearson's correlation analysis, subsequently stratified for sex and nationality. Linear regression analysis was applied in order to identify the best prediction model for identify the new daily cases, including as regressors, $\mathrm{Ct}$ values, new daily tests and age.

\section{Ethics Approval}

The study was approved by the National COVID-19 Research and ethics committee, code number: CRT-COVID2020-082.

\section{RESULTS}

\section{Characteristics of the study population}

The total number of positive cases identified between May 4, 2020 and September 30, 2020 (5-month period) was 63,879 . Mean age of the individuals was 34.02 years $( \pm 16.403)$, with a male-majority $(67.24 \%, \mathrm{n}=42953)$. Bahrainis represented majority of the cohort $(53.68 \%)$. Only $35.25 \%(n=22,519)$ of the cohort was symptomatic. The mean number of tests conducted per day was 9140 tests $( \pm 1860)$, while the mean number of newly diagnosed cases per day was $493( \pm 142)$. Mean proportion of positive tests was therefore $5.43 \%( \pm 1.29)$. The mean Ct values of the population is 25.49 (95\% CI: 25.45 - 25.52). Minimum recorded $\mathrm{Ct}$ value was 10.16 , while the maximum was 40.38 . The weighted mean Ct value in the $25^{\text {th }}$ percentile was 22 , and 28.89 in the $75^{\text {th }}$ percentile. Table 1 summarizes the sample characteristics. 
Table 1 - General characteristics of the sample population.

\begin{tabular}{|l|c|c|}
\hline \multicolumn{1}{|c|}{ Variable } & Frequency & Mean \pm SD \\
\hline Total sample & 63879 & 34.02 years $( \pm 16.403)$ \\
\hline Age & $\begin{array}{c}\text { Female }(32.8 \%)(20,926) \\
\text { Male }(67.2 \%)(42953)\end{array}$ & \\
\hline Sex & $\begin{array}{c}\text { Bahraini }(53.7 \%) \\
\text { Expats }(46.3 \%)\end{array}$ & \\
\hline Nationality & $\begin{array}{c}\text { No } 65 \%(41,360) \\
\text { Yes } 35 \%(22519)\end{array}$ & \\
\hline Symptoms & & $25.49( \pm 4.51)$ \\
\hline CT Values & & 4140 tests $( \pm 1860)$ \\
\hline $\begin{array}{l}\text { Number of } \\
\text { daily tests }\end{array}$ & & $493( \pm 142)$ \\
\hline Daily cases & &
\end{tabular}

Primary endpoint: relationship between Ct values and percentage of positive tests

Analysis of the association between $\mathrm{Ct}$ values and ratio of new daily cases to daily tests (percentage of positive tests), reveals a significant correlation coefficient of -0.06 (95\% CI: -0.06 to $-0.05, \mathrm{p}<0.001)$ (Figure 1).

When plotting standardized weekly mean Ct values against the mean weekly proportion of positive tests, a 1-week lead on positive tests (taking into consideration incubation periods) appeared most significantly correlated with Mean Ct (Figure 2). This as opposed to no lead or having a 2-week lead. In other words, a decrease in mean $\mathrm{Ct}$ values in one week, was best correlated with an increase in cases 1-week forward from that point in time.

\section{Factors affecting $\mathrm{Ct}$ value}

Analysis of the association between $\mathrm{Ct}$ values and patients' age returned a non-significant Pearson correlation ( $\mathrm{r}=0.00, \mathrm{p}=0.455)$ (Figure 3$)$. Nationality was found to be significantly associated with $\mathrm{Ct}$ value as illustrated in Figure 4a. Bahraini nationals had significantly higher mean $\mathrm{Ct}$ values than their non-Bahraini/expat counterparts (25.53 vs. 25.44, $\mathrm{p}=0.014)$. As for sex, no association was found with Ct values (Figure $4 \mathrm{~b}$ ). However, males did report a lower $\mathrm{Ct}$ value on average compared to females ( 25.48 vs. 25.510, $\mathrm{p}=0.522$ ). Finally, symptomatic status was found to be significantly associated with lower $\mathrm{Ct}$ values $(\mathrm{p}=0.001)$, when compared to asymptomatic status (24.93 vs. 25.79$)$ (Figure 5).

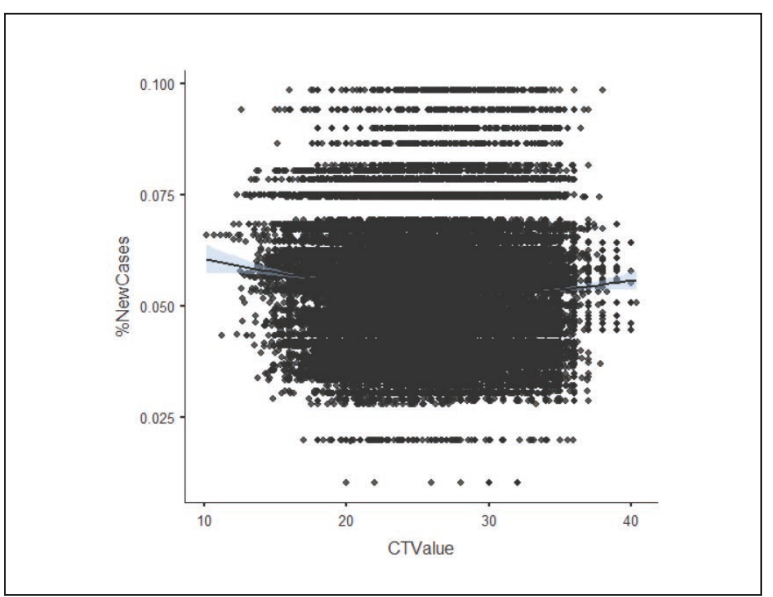

Figure 1 - Pearson's correlation between ct values and percentage of positive tests.

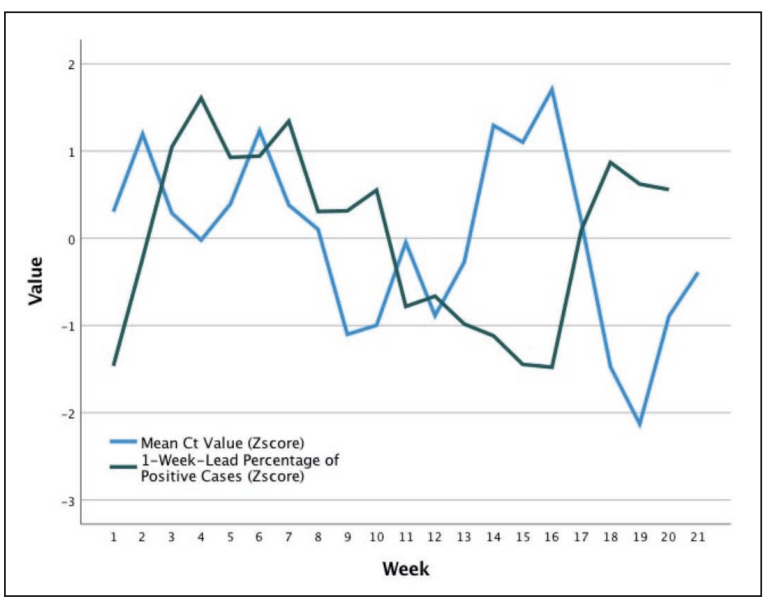

Figure 2 - Standardized Z-score of weekly mean Ct values and percentage of positive tests with 1-week lead.

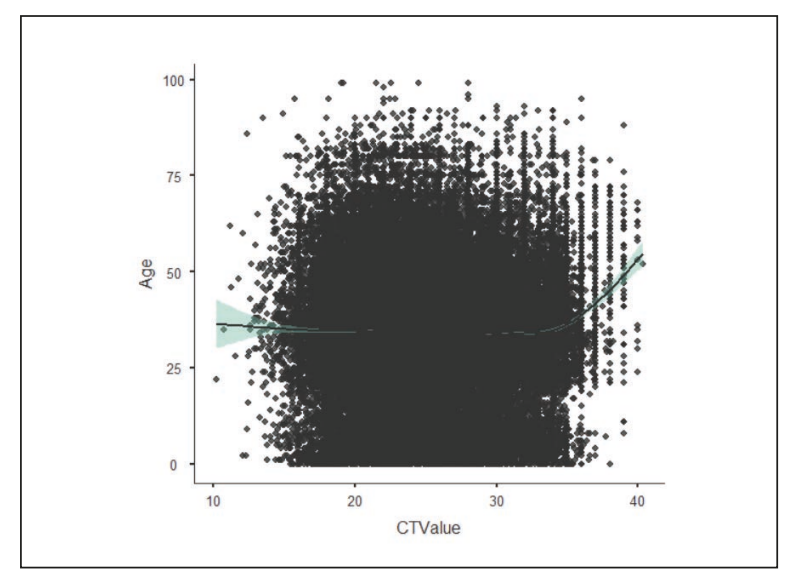

Figure 3 - Effect of age on Ct values. 
Predictive model parameter identification

A regression model on association between $\mathrm{Ct}$ values (predictor) and new daily cases (outcome) was also conducted (Table 2, Figure 1). The covariates included age and daily tests. Pearson's correlation returned a strong $\mathrm{r}$ value of 0.673 , and an $\mathrm{r}^{2}$ of 0.453 . The model's level of prediction was $45.3 \%$. Table 2 shows the regression model, where $\mathrm{Ct}$ value had a significant negative correlation with new daily cases (coef $=-1.82, \mathrm{p}<0.001)$. Daily tests were positively correlated with daily new cases (coef $=0.05$, $\mathrm{p}<0.001$ ). Age was not found to be associated with new daily cases.

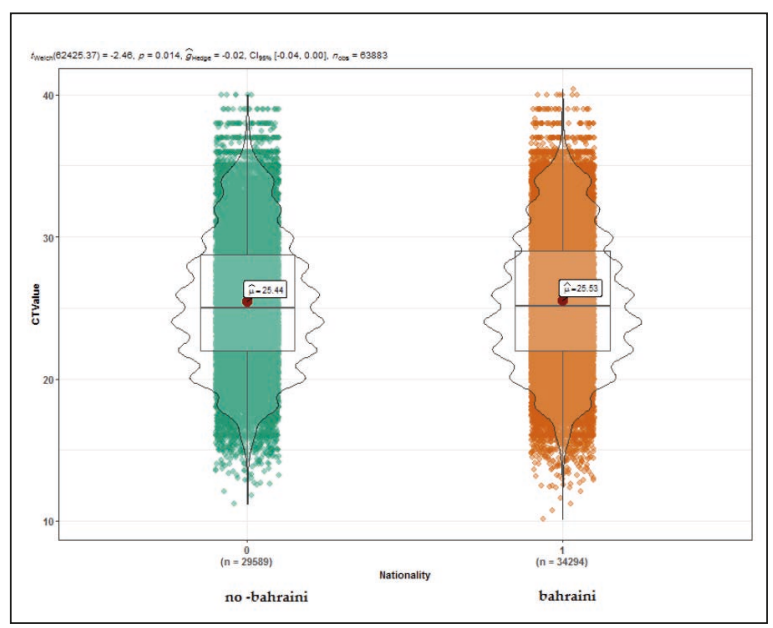

Figure 4a - Effect of nationality on Ct values.
Development of the predictive model

Based on the linear regression analysis, the equation for the prediction of the new daily cases is:

New Daily Cases $=65.88+(-1.81 \times$ Mean Ct Value $)$ $+(0.05 \times$ Mean Daily Tests $)$

When analyzing the relationship between Ct values and ratio of positive tests while stratifying for nationality, the significance of the correlation varies. A negative correlation was found between Bahraini cases $(\mathrm{p}=0.001)$ only. Additionally, when stratifying for sex, the relationship was found to be negative and significant in females $(r=-0.1$, $\mathrm{p}=0.001)$, but lower and non-significant in men $(r=-0.03, p=0.459)$ (see also Figure 6, 7a and 7b).

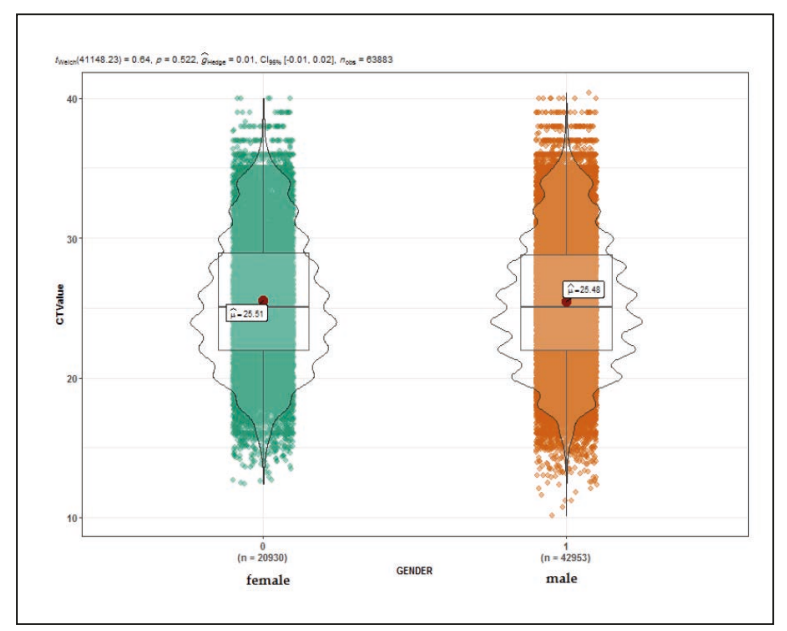

Figure 4b - Effect of sex on Ct values.
Figure 5 - Mean Ct values of symptomatic versus asymptomatic patients.

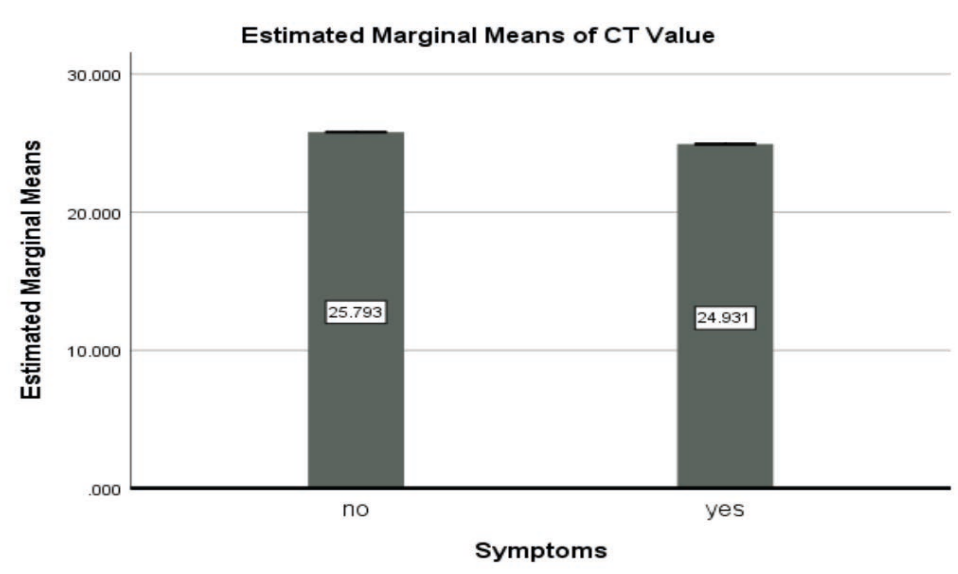

Covariates appearing in the model are evaluated at the following values: Age $=34.02$, Bahraini $=.54$, gender $=.67$, new cases ration new test daily $=.054283264526152$

Error bars: $95 \% \mathrm{Cl}$ 
Table 2 - Regression model on the association between Ct values and new daily cases. Model adjusted for regressors: Age, New Daily tests.

\begin{tabular}{|l|c|c|c|c|}
\hline \multicolumn{1}{|c|}{ 95\% Confidence Interval } & \multicolumn{2}{c|}{ Upper } & $p$ \\
\hline Predictor & Estimate & Lower & 72.29150 & 0.000001 \\
\hline CT Value & 65.88393 & 59.47637 & -1.65007 & 0.000001 \\
\hline Daily Tests & -1.81932 & -1.98857 & 0.05202 & 0.000001 \\
\hline Age & 0.05160 & 0.05117 & 0.07133 & 0.33153 \\
\hline
\end{tabular}

\section{DISCUSSION}

The correlation between RT-PCR Ct values in an infected sample of the population and a change in the overall number of positive cases over time, has not been reported in the literature to date. In this study, Ct values were used as an indicator of viral load and transmissibility. Considering that positive cases with low $\mathrm{Ct}$ values have a higher viral load than cases with high Ct values, and that a higher viral load leads to an increase in viral shedding and thus contagiousness in a population, it is reasonable to expect that a decrease in population $\mathrm{Ct}$ values would correlate with a surge in cases.

According to our findings, as hypothesized, RTPCR Ct values seemed to be negatively correlated with the number of daily cases. In other words,

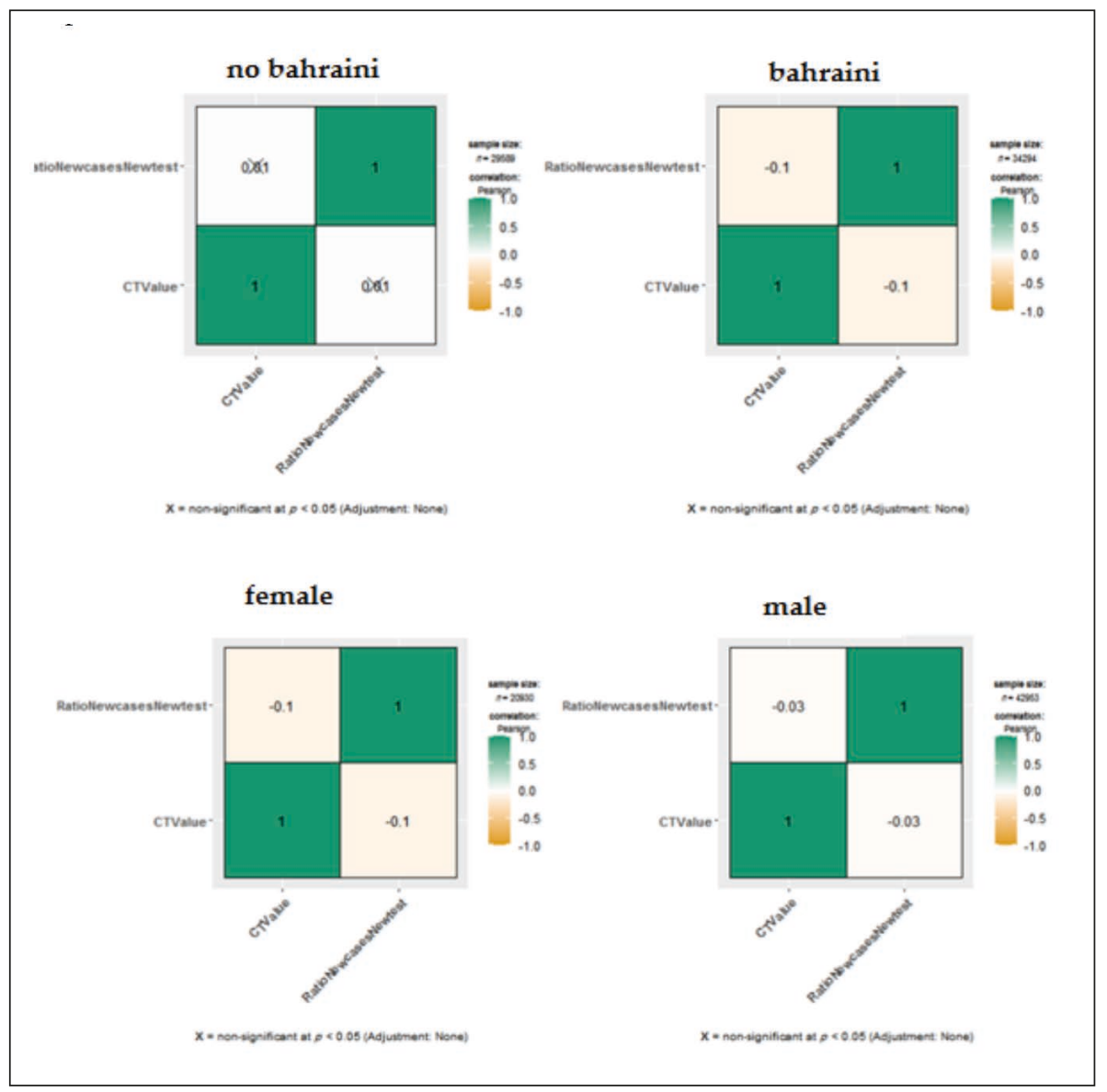

Figure 6 - Effect of nationality and sex on the relationship between $\mathrm{Ct}$ values and percentage of positive tests. 


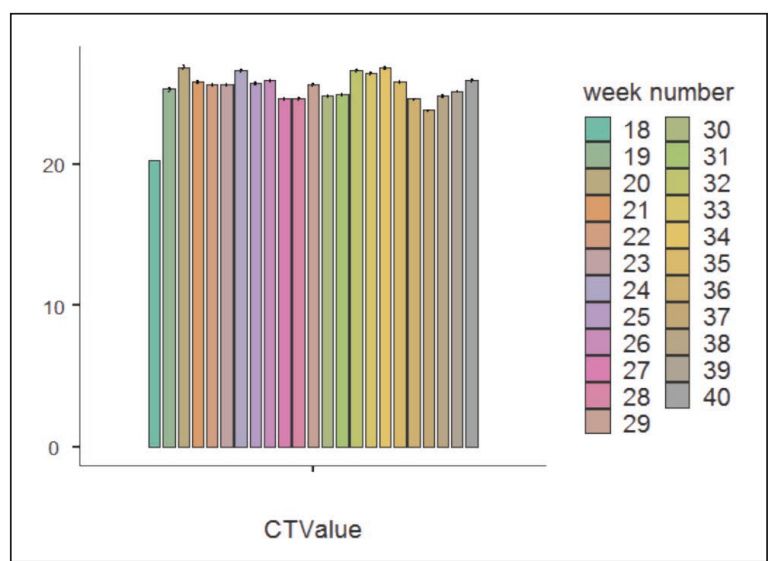

Figure 7a - Mean Ct values from week 18 to week 40.

the lower the $\mathrm{Ct}$ value, the higher the fraction of new positive tests in the population. Similarly, a recent population-based study (pre-print) from Massachusetts, USA, has identified a relationship between the population-level cross-sectional distribution of $\mathrm{Ct}$ values and the growth rate of the epidemic. The data was used to successfully develop an accurate inference model of the outbreak's trajectory [10]. A surveillance study from Italy has also reported a significant increase in $\mathrm{Ct}$ values over three different consecutive epidemic periods, indicating a possible association with underlying epidemiologic dynamics [11]. Considering evidence showing that every 1-unit increase in $\mathrm{Ct}$ values demonstrates a 0.64 odds ratio for positive viral culture (95\% CI; 0.49-0.84; $\mathrm{P}<.001$ ), it would be reasonable to infer that the association of lower $\mathrm{Ct}$ with a surge in cases is driven by increased viral infectivity in the population. In support of this hypothesis, one study from Singapore found that surface contamination from admitted patients declined with increasing $\mathrm{Ct}$ values and duration of illness. No correlation however was found between $\mathrm{Ct}$ values of clinical samples and $\mathrm{Ct}$ values of environmental samples across days of illness [12, 13].

Upon stratification by demographic factors, the association between lower $\mathrm{Ct}$ values and increase in fraction of positive tests remains the case for all ages, females, and Bahraini-nationality, but does not apply to Males and Non-Bahraini nationalities in our cohort. Possible justifications for these observations are multi-factorial. First, regarding $\mathrm{Ct}$ values not being correlated with proportion of positive tests in non-Bahrainis, this may be attributed to the unique characteristics and confounders of this sub-group and the associated targeted public health measures. The large majority of non-Bahrainis in our cohort are composed of migrant workers. A number of observations were made early on in the outbreak that made this sub-group stand out, keeping in mind the often-characteristic background of a lower socioeconomic status. Notably, it was observed that the symptom threshold at which this sub-group would present to the healthcare services was much higher than the Bahraini subgroup. Fur-
Figure 7b - Relationship between $\mathrm{Ct}$ values (adjusted for percent of new cases) throughout study period.

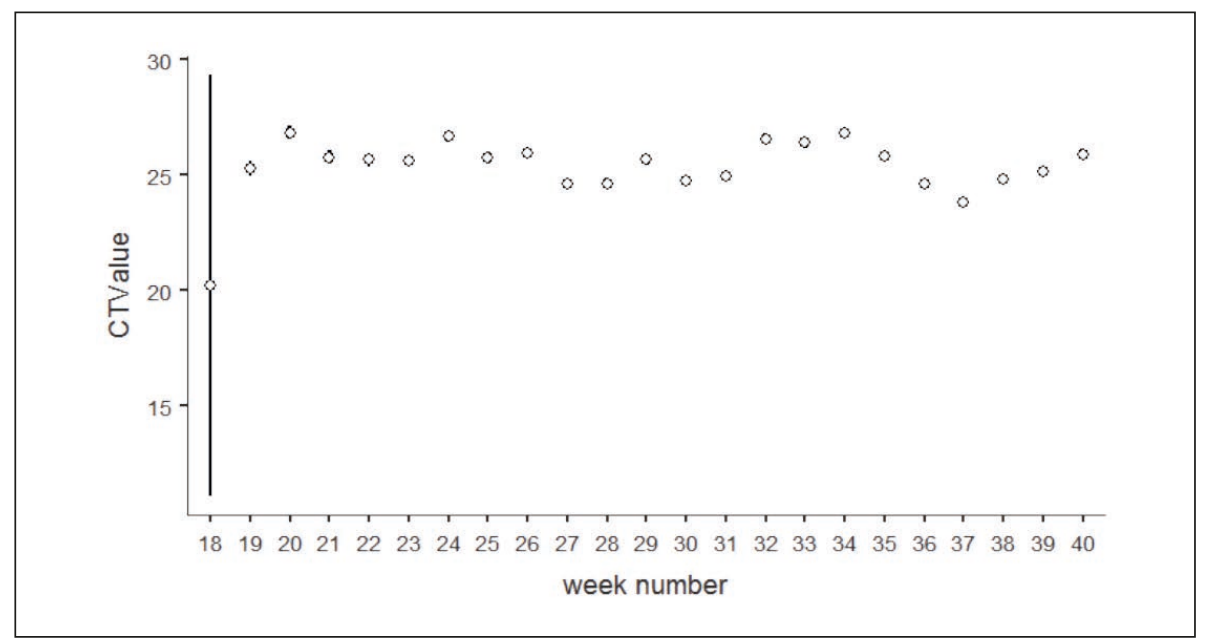


thermore, living areas of migrant workers where targeted for COVID-19 screening, often finding whole buildings of positive cases; this may have also been a major contributing factor to the statistically significant higher prevalence of low $\mathrm{Ct}$ values in this subgroup. Keeping both of these unique observations in mind, the identification of a correlation via comparison of changes in $\mathrm{Ct}$ values and number of cases may have been diluted by the skewed homogenous nature of this subgroup. Furthermore, this subgroup was exposed to unique and intensive public health campaigns that attempted to reduce the household cluttering of migrant workers and provide health education and awareness. This was largely effective, approaching a standardized state between Bahraini and non-Bahraini subgroups towards the end of the study period (which may explain the clearer negative correlation between $\mathrm{Ct}$ values and proportion of positive tests towards the second half of the study period as visually shown in Figure 2). As such, it is clear from our observations that the non-Bahraini subgroup presents with unique, multifactorial confounders and potential factors that may have affected and differentiated the association between $\mathrm{Ct}$ value and number of cases. In fact, biologic and genetic/epigenetic factors may have also been at play.

As for the finding regarding the male sex not being associated with $\mathrm{Ct}$ value and number of cases, this may be attributed to several possibilities. The first, and most likely, is that the large majority of non-Bahraini migrant workers were male, which bring us back to the confounders associated with this subgroup. Other possibilities may be related to the social and health behaviors of males, and to biological differences. The mechanism of such factors and how they may be related, if at all, remains unclear. More studies in different cohorts while accounting for confounders may help develop a fuller picture.

It is also important to consider that the mean $\mathrm{Ct}$ value for the cohort was $25.46 \pm 4.69$. As such, the $\mathrm{Ct}$ values remained largely stable, with no statistically significant weekly changes over the time period, despite changes in number of new daily cases. Meticulous contact tracing, testing, and quarantining may have contributed to maintaining the $\mathrm{Ct}$ values in the middle, with the majority being between $\mathrm{Ct} 20$ and 30. The lack of major changes may have not allowed us to view all as- sociations between $\mathrm{Ct}$ values and positive cases to the extent to which they may be intertwined in reality, which may have surfaced at a specific threshold or degree of difference. On the other hand, it is also important to consider that the time between testing and acquiring of infection varies among individuals. Therefore, a patient may have acquired a COVID-19 infection during a period of low $\mathrm{Ct}$ values in the population clusters, but was only tested positive a while after, when the corresponding $\mathrm{Ct}$ values had increased.

As for the other findings in our study, the reported male to female sex ratio was 2.05:1. This ratio is much higher than the ratio of 1.03:1, reported by the WHO's global case-based surveillance system [14]. This is mainly due the fact that the $47 \%$ of infected cases during the studied period were Non-Bahraini, with majority of whom belong to labor camps, which have a male majority. Moreover, this may also be driven by cultural norms, with males more likely to socially mix outdoors and thus be infected. This may also be related to the higher percentage of males in the population $(62.6 \%)$, with a male to female ratio of 1.67:1 [15].

Of the cohort, only $35.25 \%$ were symptomatic, with $64.75 \%$ presenting asymptomatically, one of the highest asymptomatic rates reported in literature to date [16]. The symptom status for these cases was documented upon testing and upon triage; hence a subset of the asymptomatic cases might be further classified as pre-symptomatic. The prevalence of asymptomatic patients is very difficult to assert, a meta-analysis of 50,155 cases from 41 studies suggests that it may account for $15.6 \%$ (95\% CI, $10.1 \%-23.0 \%)$ of SARS-CoV-2 infections [16]. The prevalence reported in our cohort may be higher for a number of reasons, including more open screening policies and comprehensive contact tracing. It may also be associated with other factors inherent to cohort characteristics, such as age (which is on the low-end of reported means) and comorbidities [17]. It is worth noting that our study found a correlation between lower $\mathrm{Ct}$ values and symptomatic presentation, however the mean $\mathrm{Ct}$ value difference between symptomatic and asymptomatic was 0.86 which may not be a clinically significant difference. It may be suggested that a higher asymptomatic prevalence could be indicative of more well-controlled isolation measures leading to a lower viral transmis- 
sion in the population. It must be noted however that since this is a cross-sectional study of a specific point in time, that some of the cases may be pre-symptomatic rather asymptomatic. Regardless, the prevalence would still be higher than that reported in other cohorts [16]. Furthermore, higher Ct values being correlated with asymptomatic presentations may be attributed to a pathophysiologic mechanism whereas a lower viral load may decrease or dampen the symptomatic presentation of said infected patient. It is important to also consider however that asymptomatic individuals may have had higher $\mathrm{Ct}$ values due to being identified at a later time since infection compared to symptomatic individuals. It has been reported that $\mathrm{Ct}$ values increases over illness duration [18]. Although lower $\mathrm{Ct}$ values indicate a higher viral load and thus increased infectiousness, which would be expected to be associated with a surge in cases as was shown in our data, it is important to consider the confounding nature of symptomatic status. For one, asymptomatic status can lead to increased transmission compared to symptomatic, because symptomatic individuals are able to identify themselves self-isolate and get tested as soon as possible. Since asymptomatic cases also had statistically significant higher Ct values according to our findings, it is important to consider that the association between $\mathrm{Ct}$ values and infectivity on a population scale may be even more significant than reported. Furthermore, all COVID-19 cases had their Ct values reported to the COVID-19 taskforce. Therefore, those with lower $\mathrm{Ct}$ values might have had more aggressive tracing and management, which can indirectly contribute to lowering infectivity from that individual.

\section{Clinical and public health implications}

Change in COVID-19 cases is largely multifactorial, affected by a number of factors that include testing policies, public health measures, population behavior and compliance, religious/cultural events/holidays, reopening strategies and viral factors [19]. For these reasons, $\mathrm{Ct}$ values alone may not be useful in predicting a change in COVID-19 cases, despite our study showcasing a moderate association between these variables. However, they may still be of benefit as part of a multivariable model: especially one that takes into consideration individual characteristics (e.g. symptomatic status, demographic conditions) as related to individual behavior, and population characteristics (e.g. public health policies) as related to population behavior (e.g. compliance to regulations).

Regardless, whether these findings would be clinically relevant is debatable. This reaffirms that disease progression is multifactorial. However, it may be more useful as a metric to use as part of a more diverse tool. Additionally, since healthcare professionals interact with infected patients, extra precautions may be justified in the case of patients with lower $\mathrm{Ct}$ values, as they would shed more virus particles and are more likely to be more infectious [20]. Additionally, this may help with deciding which patients are better isolated or placed in negative pressure rooms. The main applications of $\mathrm{Ct}$ values however would be best concentrated as epidemiologic/public health and forecasting efforts.

From a public health perspective, knowing that average viral loads may be associated with surge in cases and with a more symptomatic presentation, supports the importance of decreasing viral exposure and the gradual nature of infection. This is in contrast to adopting an "all-or-none" attitude of completely eliminating potential exposure. For example, this would translate to emphasizing that the more socially distanced people are, the lower the chance of acquiring COVID-19, as opposed to 6-feet social distancing being reiterated. This may especially be of importance in public campaigns, reinforcing the importance of maximizing distance and minimizing contact and interaction, rather than only reiterating specific instructions for what should and should not be done to avoid contacting COVID-19.

\section{Limitations}

Despite this being one of the largest cohorts on COVID-19 in the region, and the largest to investigate $\mathrm{Ct}$ values in relation to case trajectory, the limitations are important to be addressed. To begin with, although this is a multi-center analysis, it remains a national one in a small country. National public health measures and screening policies differ from one place to the other, which necessitates the need for a global cohort when investigating the macro-behaviors and characteristics of COVID-19. The Ct values as reported in our study have been fairly stable throughout the fivemonth period from a statistical standpoint, which 
would make it non-ideal to test for associations between $\mathrm{Ct}$ values and case trajectory. For that reason, longer periods or cohorts from different populations may be more suitable to investigate an association. Additionally, a linear regression may not be the most suitable statistical approach to model the data; however, the aim of the pilot study was to explore the correlations using initially simple models. Screening policies additionally play a vital role in determining the cohort characteristics. Our study involved cases from varying screening approaches, including admitted patients, symptomatic suspected cases in outpatient setting, contact tracing, population screening in case of travel, random testing in the community and individual-requested testing. In order to minimize bias and confounding factors, ideally only cohorts from randomized population screening should be included. This would also help control the number of tests conducted per day. The different frequencies of testing may have impacted the proportion of positive cases identified per day, contributing to the change in cases in a way that conflicts with Ct values as the intended independent variable. Additionally, public health measures changed reactively throughout the study period, which likely impacted the transmission of the virus in the population in a way that would be difficult to account for.

Furthermore, human bias in tracking down the cases with lower $\mathrm{Ct}$ values may have skewed the findings. Additionally, the $\mathrm{Ct}$ values can vary depending on how the sample has been collected. For instance, a poorly collected nasopharyngeal swab can have very high $\mathrm{Ct}$ values, thus leading to biased findings regarding infectiousness or disease severity. $\mathrm{Ct}$ values are also determined by the technical competence of the person performing the test, calibration of equipment, and analytical skills of the interpreters, all of which could have impacted the results. Furthermore, inconsistency in Ct values can result from irregular amplification efficiency which has been observed even when using the same commercial kit; hence, it is recommended to locally determine the limit of detection (LoD) of our tests. To add to that, double delta Ct values were not used in our study, which would have been a more standardized method to report viral loads across different PCR kits and machines. The retrospective observational study design is also in itself an important limitation.
Time from symptom onset is also an important variable that could be a confounding factor, however the data on it was difficult to collect. Other confounding variables influencing both $\mathrm{Ct}$ values and newly identified cases were difficult to completely account for in the analysis.

In conclusion, the spread of a viral disease of the like of COVID-19 in a population is multifactorial. Public health measures, testing protocols, population compliance, and viral factors all play a role in the degree of transmission, rate of positive cases, and epidemiologic trajectory. Understanding the correlations of a simple, commonly available measure like $\mathrm{Ct}$ values with epidemiologic variables and patient demographics may pave the road for the development of tools to forecast a pandemic's behavior. Our first-of-its-kind and largest-to-date retrospective multicenter national study points towards a possible correlation between $\mathrm{Ct}$ values and the ratio of positive tests in a population. Additionally, symptomatic status was associated with a lower $\mathrm{Ct}$ value. This reaffirms the need to develop multivariable models to forecast viral pandemics, possibly including $\mathrm{Ct}$ values. Similar studies of a large magnitude in different countries may be able to shed more light on this topic.

\section{Conflict of interest disclosures:}

The authors declare no conflict of interest

\section{Funding}

None

Ethics approval and consent to participate: The study was approved by the National COVID19 Research and Ethics Committee.

\section{Author contributions}

AKA, AIA collected the data. AKA, MQ, EJ contributed to the design of the study. SP performed the statistical analysis. SM scripted the first draft. All author contributed to writing the manuscript. All authors have read and approved the final manuscript.

\section{REFERENCES}

[1] Tang S, Mao Y, Jones RM, et al. Aerosol transmission of SARS-CoV-2? Evidence, prevention and control. Environ Int. 2020; 144: 106039. 
[2] World Health Organization. Transmission of SARSCoV-2: implications for infection prevention precautions: scientific brief, 09 July 2020. World Health Organization; 2020.

[3] Thermo Fisher Scientific. Real-Time PCR: Understanding Ct Application Note. 2016. [Available at: https://www.thermofisher.com/bh/en/home/life -science/pcr/real-time-pcr/real-time-pcr-learningcenter/real-time-pcr-basics / real-time-pcr-understanding-ct.html].

[4] Bullard J, Dust K, Funk D, et al. Predicting infectious SARS-CoV-2 from diagnostic samples. Clin Infect Dis. 2020; 71 (10), 2663-6

[5] La Scola B, Le Bideau M, Andreani J, et al. Viral RNA load as determined by cell culture as a management tool for discharge of SARS-CoV-2 patients from infectious disease wards. Eur J Clin Microbiol Infect Dis. 2020; 39 (6), 1059.

[6] Hiscott J, Alexandridi M, Muscolini M,et al. The global impact of the coronavirus pandemic. Cytokine Growth Factor Rev. 2020; 54, 1-9.

[7] Emanuel EJ, Persad G, Upshur R, et al. Fair Allocation of Scarce Medical Resources in the Time of Covid-19. N Engl J Med. 2020; 282 (21), 2049-55.

[8] Centres for Disease Control and Prevention. Coronavirus Disease 2019 (COVID-19). 2020. [Available at: https: / / www.cdc.gov/coronavirus/2019-ncov/cases-updates / forecasts-cases.html].

[9] Roser M, Ritchie H, Ortiz-Ospina E, et al. Coronavirus pandemic (COVID-19). Our World in Data. 2020. [Available at: https://ourworldindata.org/coronavirus].

[10] Hay JA, Kennedy-Shaffer L, Kanjilal S, et al. Estimating epidemiologic dynamics from single cross-sectional viral load distributions. medRxiv. 2020 Jan 1.

[11] Veronesi L, Colucci ME, Pasquarella C, et al. Viro- logical surveillance of SARS-CoV-2 in an Italian northern area: comparison of Real Time RT PCR cycle threshold $(\mathrm{Ct})$ values in three epidemic periods. Acta Biomed. 2020; 91, 19-21.

[12] Bullard J, Dust K, Funk D, et al. Predicting infectious severe acute respiratory syndrome coronavirus 2 from diagnostic samples. Clin Infect Dis. 2020; 71 (10), 2663-6.

[13] Chia PY, Coleman KK, Tan YK, et al. Detection of air and surface contamination by SARS-CoV-2 in hospital rooms of infected patients. Nat Commun. 2020; 11 (1), 1-7.

[14] World Health Organization. Coronavirus disease 2019 (COVID-19): situation report, 89.

[15] Countrymeters. Bahrain population (2020) live. 2020. [Available at: https:/ / countrymeters.info/en/Bahrain].

[16] He J, Guo Y, Mao R, Zhang J. Proportion of asymptomatic coronavirus disease 2019: A systematic review and meta-analysis. J Med Virol. 2021; 93 (2), 820-30.

[17] Khalili M, Karamouzian M, Nasiri N, Javadi S, Mirzazadeh A, Sharifi H. Epidemiological Characteristics of COVID-19: a systemic review and meta-analysis. MedRxiv. 2020 Jan 1.

[18] Singanayagam A, Patel M, Charlett A, et al. Duration of infectiousness and correlation with RT-PCR cycle threshold values in cases of COVID-19, England, January to May 2020. Eurosurveill. 2020; 25 (32), 2001483. [19] Hammad OA, Alnazzawi A, Borzangy SS, et al. Factors influencing global variations in COVID-19 cases and fatalities; A Review. Healthcare (Basel, Switzerland). 2020; 8 (3), E216.

[20] Rahman AK, Al Ali S, Yaghi O, Otoom S, Atkin SL, Al-Qahtani M. The cycle of threshold measurement for COVID-19 infection and its implications in patient's length of hospital stay. Saudi J Pathol Microbiol. 2020. In Press. 